\title{
KINEMATICS OF OXYGEN-RICH FILAMENTS IN PUPPIS A
}

\author{
P. Frank Winkler, John H. Tuttle \\ Middlebury College, Middlebury, VT, U.S.A.
}

Robert P. Kirshner

Harvard-Smithsonian Center for Astrophysics, Cambridge, MA, U.S.A.

Michael J. Inwin

Institute of Astronomy, Cambridge University, Cambridge, U.K.

\begin{abstract}
We have measured proper motions for fast, oxygen-rich knots in Puppis A, which we demonstrate are probably uncontaminated ejecta from the progenitor star's core. Typical fast knots show motions of 0.1-0.2 arcsec $\mathrm{yr}^{-1}$ diverging from a point $4^{\prime}$ northeast of the center of the radio shell. A model assuming constant expansion fits the data well and gives an age of $3700 \pm 300 \mathrm{yr}$ for Puppis A. We also present new spectra which indicate the presence of neon along with oxygen in the fast knots.
\end{abstract}

\section{Introduction}

Puppis A was one of the first supernova remnants (SNRs) in which optical nebulosity was identified (Baade and Minkowski 1954), and the chaotic morphology of its optical filaments has long been puzzling. The spectra of the brightest filaments indicate a high abundance of nitrogen (Dopita, Mathewson, and Ford 1977) and modest radial velocities: $<300 \mathrm{~km} \mathrm{~s}^{-1}$ (Elliott 1979; Shull 1983). In striking contrast are the faint filaments discovered by Winkler and Kirshner (1985) which are extremely rich in oxygen and have radial velocities up to $1500 \mathrm{~km} \mathrm{~s}^{-1}$. Winkler and Kirshner interpreted these fast filaments as vestiges of ejecta from the core of the supernova star, similar to the fast-moving knots in Cas A. This picture meshes with the X-ray spectra of Puppis A, which indicate a hot plasma heavily enriched in oxygen and neon (Canizares and Winkler 1981). A few solar masses of ejecta similar in composition to the fast-moving optical filaments could have provided the enrichment inferred from the X-ray plasma.

The near absence of hydrogen in the fast filaments of Puppis A suggests that they have interacted rather little with interstellar or circumstellar material, and thus that they may remain relatively undecelerated since the supernova event. Our proper-motion study has enabled us to measure the expansion of the fast filament system and to determine the kinematic age of Puppis A to be $3700 \pm 300 \mathrm{yr}$. The, evolution of SNRs and their interaction with the interstellar medium is now a sufficiently mature subject to merit its own IAU Colloquium, yet only a handful of the youngest remnants have well-established ages. The measurement based on kinematics inducts Puppis $A$ as the senior member into this heretofore exclusively youthful society.

\section{Astrometry and the Expansion Model}

Our measurements are based on 4-m prime-focus plates taken from CTIO at three epochs: 1978.2, 1984.1, and 1986.2. Plates were taken in two broad wavelength bands, IIlaJ + GG495 ("green", 490-530 nm) and IIlaF + RG610 ("red", 610-700 nm), of two overlapping fields covering virtually the entire Puppis A remnant, which has a diameter of about $55^{\prime}$. The ejecta-rich filaments are easily seen on the green plates due to their strong [O III] emission, but are virtually invisible on the red plates. For this study we have used five green plates, which include three pairs with identical centers and baselines of 5.9 years or more.

We used a digital technique to carry out the astrometry. The plates were scanned and digitized using the Automatic Plate Measuring System (APM) at the Institute of Astronomy, Cambridge (Kibblewhite et al .1984). The pixel size of $30 \mu \mathrm{m}$, equivalent to $0 " .565$ at the plate scale of 18.6 arcsec $\mathrm{mm}^{-1}$, resulted in 30 million pixels per plate. 
Measurement of the displacement of an individual knot between a reference $(R)$ and a comparison (C) plate from different epochs is accomplished by first selecting a region about $2^{\prime} \times 2^{\prime}$ in size surrounding the knot. Centroids are determined for all the features with stellar profiles; the optimum translation and rotation of the $\mathrm{C}$ plate to bring its stars into alignment with those on the $\mathrm{R}$ plate is calculated; and the $C$ region is then rebinned coincident with the $R$ one.

A knot to be measured is then selected interactively with a cursor; ideally the knot should be "bright," well defined, and have no stars within the cursor-defined area. The displacement of the filament on plate $C$ relative to plate $R$ is determined using a statistic that minimizes the absolute value of the difference between pixel values on the two plates, summed over the knot area. This statistic is more robust than a least-squares one, and leads to uncertainties of $0 " .1-0 " .25$ in each component of displacement from a single plate pair.

We measured displacements for 11 knots on each of the three pairs of plates, one pair separated in epoch by $5.9 \mathrm{yr}$, the other two pairs by $8.0 \mathrm{yr}$. The 11 knots have proper motions in the range $0 " .09-0 " .22 \mathrm{yr}^{-1}$. All the measured knots are shown with vectors indicating their proper motions in Figure 1.

We have fit the data with a model which assumes the undecelerated expansion of all the filaments from a common origin in space and time. This model has only three parameters: the $x$ and $y$ coordinates of the expansion center and the age of the remnant. The model gives a good fit to the data, with a $\chi^{2}{ }_{v}$ value of 1.4 . Although the ejecta-rich filaments are distributed over a sector of only $70^{\circ}$, the best-fit parameters are nevertheless well determined:
Age $=3700 \pm 300 \mathrm{yr} ;$
Expansion Center:
R.A. $=8^{\mathrm{h}} 20^{\mathrm{m}} 44^{\mathrm{s}} .3$
Dec. $=-42^{\circ} 47^{\prime} 48^{\prime \prime}$

The expansion center is located 4' northeast of the center of the radio shell. The error ellipse is shown in Figure 1, and all the proper motion data are plotted in Figure 2, along with the best-fit model.

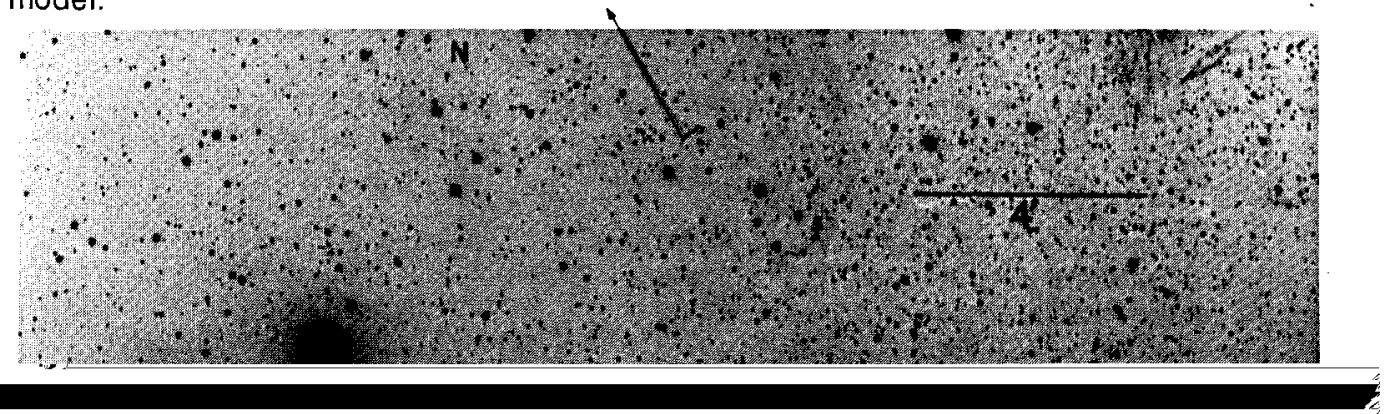



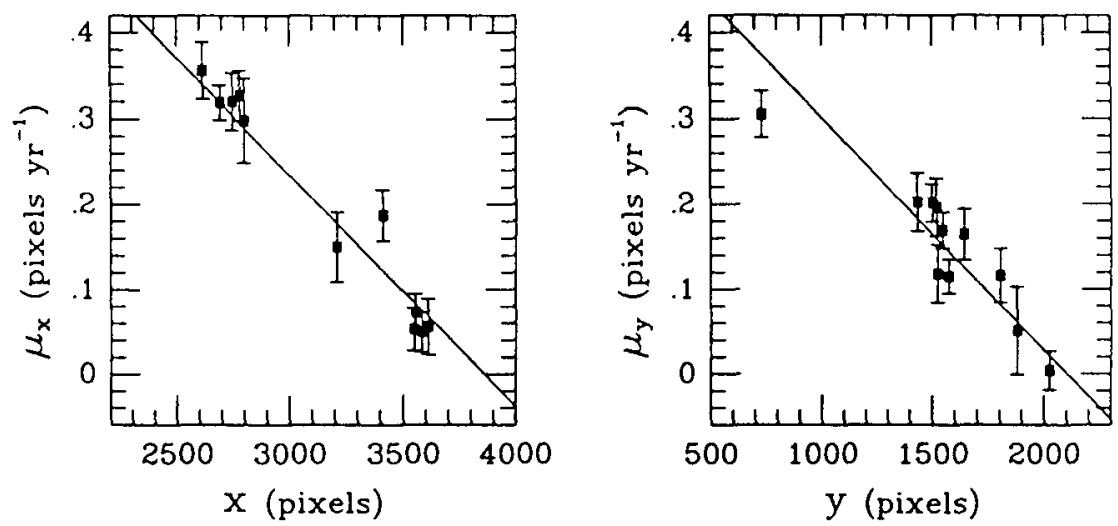

Figure 2. $X$ and $Y$ components of proper motion vs. position; the line is the best-fit free expansion model.

\section{Spectra and Radial Velocities}

Through spectroscopy with the CTIO 4-m telescope, we have extended the spectra shown in Winkler and Kirshner (1985) to shorter wavelengths and to additional filaments. The spectrum of the omega filament shown in Figure 3 is typical; it may be compared with the longer wavelength spectrum shown in Figure 2 of Winkler and Kirshner. It is particularly interesting to note the presence of neon, indicated by [Ne III] $\lambda \lambda 3869,3967$. These lines provide further evidence that the fast-moving filaments are similar in composition to the material which enriched the Puppis $A$ $\mathrm{X}$-ray plasma, where strong lines of $\mathrm{Ne} \mathrm{IX}$ and $\mathrm{Ne} X$ indicate an overabundance of neon as well as oxygen (Canizares and Winkler 1981).

All of the filaments for which oxygen lines dominate the spectra show radial velocities $400<|\mathrm{vr}|<$ $1600 \mathrm{~km} \mathrm{~s}^{-1}$, most of them blue-shifted. There is little systematic relationship between radial velocity and proper motion. This is not an inconsistency; it merely means that the knots we are seeing have a range of space velocities, and presumably lie at different distances from the center. While we have not been able to use kinematics to improve the distance measurement to Puppis $\mathrm{A}$, at a distance of $2 \mathrm{kpc}$ the transverse and radial velocities of the fast filaments are comparable. Further details of the spectroscopy and the proper motions will appear in Winkler, Kirshner, and Irwin (1988).

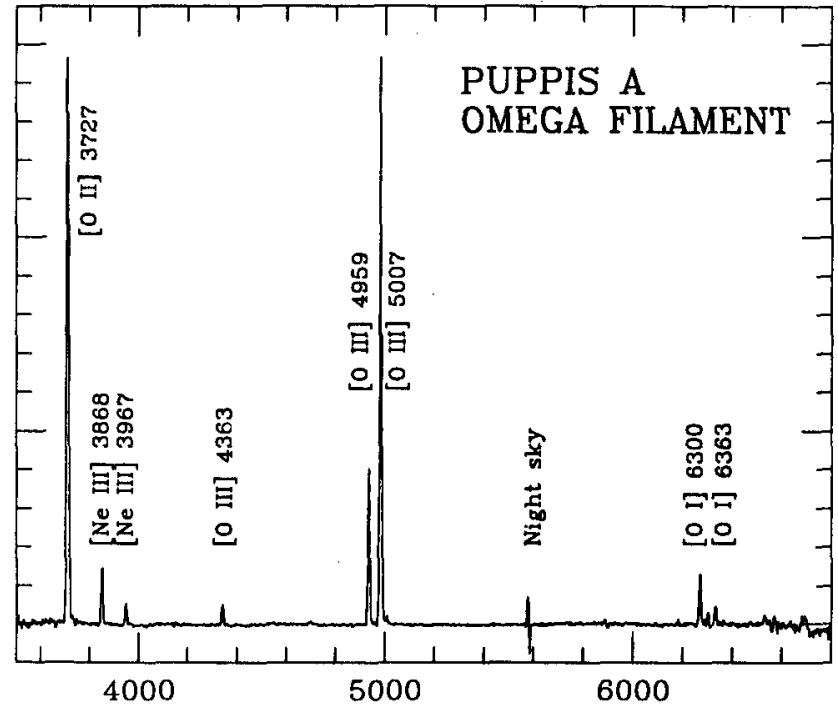

Figure 3. Spectra of the fastmoving filaments indicate a composition rich in oxygen and neon, and almost devoid of hydrogen. 


\section{Origin and Survival of Ejecta Knots}

In view of their unusual composition, high velocities, and common center of expansion, the case that the fast knots are supernova ejecta seems secure. Their space velocities are $1500-3000 \mathrm{~km}$ $\mathrm{s}^{-1}$, lower than those typically associated with Type II supernovae. But since today's fast knots represent material from the inner layers of the progenitor star, they were probably laggards at the time of the explosion. Indeed, they have managed to survive only because they are moving into the low-density shell swept out by the faster-moving supernova blast wave.

We may consider the knots as high-density bullets moving out through a low-density surround. One obvious criterion for knot survival is that the bullet can encounter no more than its own mass in ambient material without being ablated into oblivion, but Nittmann, Falle, and Gaskell (1982) have determined a more stringent criterion by considering dynamic instabilities of the bullet. As shown in Winkler, Kirshner, and Irwin (1988), knots larger than 0.1 pc with density 200 O-atoms $\mathrm{cm}^{-3}$ (typical of the minimum dimension and density of the observed knots) could reach their present distances through a surround of density $<3 \mathrm{~cm}^{-3}$.

\section{The Age of Puppis A}

Previous estimates of Puppis A's age have used the X-ray spectrum to determine the shock velocity and then used a Sedov model to obtain the age. Values in the range 4000-8000 yr have typically been obtained (Culhane 1977). Uncertainties involving lack of resolution in the X-ray spectra, non-equilibrium ionization effects, the applicability of the Sedov model, and the distance to Puppis $A$ all attend the interpretation of the X-ray data. Thus the direct kinematic measurement obtained here is probably much more reliable.

It is interesting to note that an age of only 3700 years puts Puppis A's supernova within the range of recorded human history: 2000-1400 BC based on constant expansion, and possibly somewhat younger if some deceleration has occurred. The Sumerians and Babylonians made note of astronomical events, so it is conceivable that some mention of a bright southern star may be found on cuneiform tablets. If we assume a typical peak luminosity for Type II supernovae of $M_{V}=-18, a$ distance of $2 \mathrm{kpc}$, and $A_{V}=1.5$, we may estimate the peak brightness of the Puppis $A$ event at $V \approx-5$, surely bright enough to attract notice.

We congratulate the organizers of IAU Colloquium 101 for an exceedingly well-organized meeting in which it was a pleasure to participate. We would like to thank the CTIO staff for their unflagging and good-natured support during the several observing runs necessary to obtain the plates for this project, and the Institute of Astronomy and SERC for making time available on the APM system. Financial support has come through NSF grants AST-8520557, AST-8516537, and NASA grant NAG-8389.

\section{References}

Baade, W., and Minkowski, R. 1954, Ap. J., 119, 206.

Canizares, C.R., and Winkler, P.F. 1981, Ap. J. (Letters), 246, L33.

Culhane, J.L. 1977, in Supernovae, D.N. Schramm, ed. (Dordrecht: D. Reidel), pp. 2951.

Dopita, M.A., Mathewson, D.S., and Ford, V.L. 1977 Ap. J., 214, 179.

Elliott, K.H. 1979, Mem. della Soc. Astr. Italiana, 49, 477.

Kibblewhite, E., Bridgeland, M., Bunclark, P., Cawson, M., and Irwin, M. 1984, in Astronomy with Schmidt-Type Telescopes, M. Capaccioli, ed. (Dordrecht: D. Reidel), 89.

Nittmann, J., Falle, S.A.E.G., and Gaskell, P.H. 1982, M.N.R.A.S., 201, 833.

Shull, P., Jr. 1983, Ap. J., 269, 218.

Winkler, P.F., and Kirshner, R.P. 1985, Ap. J., $299,981$.

Winkler, P.F., Kirshner, R.P., and Irwin, M.J. 1988, in preparation for Ap. J. 